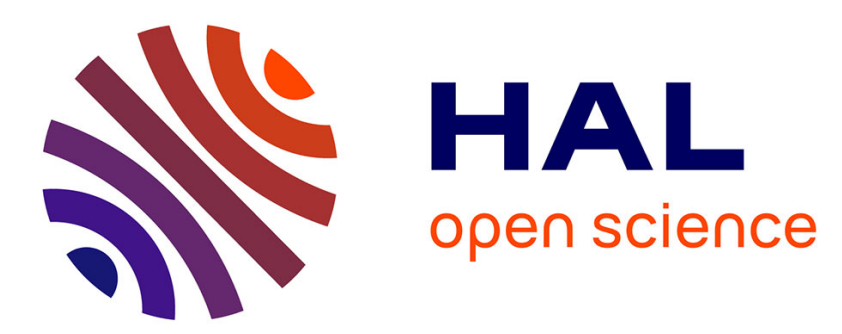

\title{
An improved 2D subdomain model of squirrel cage induction machine including winding and slotting harmonics at steady state
}

Emile Devillers, Jean Le Besnerais, Thierry Lubin, Michel Hecquet, Jean-Philippe Lecointe

\section{To cite this version:}

Emile Devillers, Jean Le Besnerais, Thierry Lubin, Michel Hecquet, Jean-Philippe Lecointe. An improved 2D subdomain model of squirrel cage induction machine including winding and slotting harmonics at steady state. IEEE Transactions on Magnetics, 2018, 10.1109/TMAG.2017.2782222 . hal-01679361

\section{HAL Id: hal-01679361 \\ https://hal.science/hal-01679361}

Submitted on 9 Jan 2018

HAL is a multi-disciplinary open access archive for the deposit and dissemination of scientific research documents, whether they are published or not. The documents may come from teaching and research institutions in France or abroad, or from public or private research centers.
L'archive ouverte pluridisciplinaire HAL, est destinée au dépôt et à la diffusion de documents scientifiques de niveau recherche, publiés ou non, émanant des établissements d'enseignement et de recherche français ou étrangers, des laboratoires publics ou privés. 


\title{
An improved 2D subdomain model of squirrel cage induction machine including winding and slotting harmonics at steady state
}

\author{
Emile Devillers ${ }^{1,2}$, Jean Le Besnerais ${ }^{2}$, Thierry Lubin ${ }^{3}$, Michel Hecquet ${ }^{1}$, Jean-Philippe Lecointe ${ }^{4}$ \\ ${ }^{1}$ L2EP, Univ. Lille, Centrale Lille, Arts et Metiers ParisTech, HEI, EA 2697, F-59000 Lille, France \\ ${ }^{2}$ EOMYS ENGINEERING (www.eomys.com), F-59260 Lille-Hellemmes, France \\ ${ }^{3}$ GREEN, Univ. Lorraine, EA 4366, F-54500 Vandœuvre-lès-Nancy, France \\ ${ }^{4}$ LSEE, Univ. Artois, EA 4025, F-62400 Béthune, France
}

\begin{abstract}
This paper proposes an improved subdomain model of squirrel cage induction machines with imposed stator currents. This new model enables to accurately compute electromagnetic quantities such as air gap flux density, instantaneous torque and forces, electromotive force including all harmonic content. The first improvement is to explicitly account for rotor motion with time-stepping technique. The second improvement consists in modelling the skin effect in rotor bars by considering each space harmonic of the stator magnetomotive force separately. Eddy-currents in rotor bars are therefore "skin-limited" and not "resistance-limited". The results are then validated with linear transient finite element analysis for both no-load and load cases, taking a topology of squirrel cage machine already used in previous references. The time and spatial harmonic content of all electromagnetic quantities is also validated by comparison with analytical expressions. The computation time is a hundred times lower than finite element as it does not require achieving a numerical transient first and the resolution for a time step is shorter. Thanks to its computational efficiency and intrinsic mesh insensitivity, this method is particularly suited to magnetic forces computation and vibroacoustic analysis of squirrel cage induction machines.
\end{abstract}

Index Terms-Magnetic field, Electrical machines, Analytical model, Harmonic analysis.

\section{INTRODUCTION}

$\mathrm{T}$ HE SubDomain Method (SDM), also called Fourier-based Model or Harmonic Model, is a semi-analytical method which has been recently developed to compute the twodimensional air gap flux density in electrical machines as well as other machine characteristics, such as electromagnetic torque, electromotive force, magnetic losses, Maxwell forces for noise and vibrations analysis. The method is based on the analytical resolution of the Maxwell equations in the air gap, stator and rotor slots using Fourier series expansion under several assumptions [1], such as the infinite permeability of iron cores. For the same modeling assumptions, SDM is as accurate as Finite Element Method (FEM) and much faster, which makes it interesting during the first design steps of electrical machines [2] or to quickly estimate magnetic forces involved in magnetic noise generation [3], [4].

SDM has been first dedicated to the modelling of various Permanent Magnet Synchronous Machines (PMSM), for which many references can be found in the literature [5], [6], [7]. As shown in [2], there are fewer references concerning Squirrel Cage Induction Machines (SCIM) [8], [9], [10], due to the difficulty of estimating eddy-currents in rotor bars and resulting rotor reaction field.

In [8], the authors developed a subdomain model to compute eddy-currents in rotor bars generated with a current sheet located at the slotless stator bore. There is actually one current sheet for each space harmonic of the stator magnetomotive force (mmf). In the rotor referential, each space harmonics rotates at a different speed and so induces eddy-currents in bars at a specific frequency. These bar currents are computed by solving the Helmholtz equation - diffusion equation at a single frequency - in the rotor slots subdomains. The total electromagnetic quantities (such as flux density) are obtained by solving independently the problem in the rotor referential for each space harmonics and summing all the contributions. This enables to get the rotor reaction field directly in the air gap flux density and to take into account the proper skin effect for each space harmonic, meaning that eddy-currents are "skin-limited".

The SCIM subdomain model presented in [9] computes the global performances of the SCIM, with both current and voltage inputs by using a single phase Electric Equivalent Circuit (EEC). Furthermore, this model enables to account for space harmonics due to both stator slotting and $\mathrm{mmf}$, by including stator slots subdomains with current density as it has been done for subdomain model PMSM [7]. As in [8], rotor bar currents are obtained by solving the Helmholtz equation. In the stator subdomains and in the air gap, the electromagnetic quantities are assumed to vary at the supply frequency, while they vary at the slip frequency in the rotor subdomains. Current harmonics in rotor bars due to the space harmonics of the stator mmf are therefore neglected. Besides, the method to account for rotor motion and especially to get the time harmonics in the air gap flux density due to rotor slotting is not specified. Concerning the computation time, the authors say that it is almost as long as FEA (3.24 minutes for their SDM against 3.58 minutes for FEA with a very fine mesh). Furthermore, the spatial flux densities results are quite inaccurate compared with those obtained using FEA. This model has been extended in [11] for the study of multi-phases SCIM under fault conditions but the subdomain modelling technique in itself is still the same as in [9].

In [10], the subdomain model also includes both rotor and stator slots subdomains. The problem is first solved without 
considering that the rotor bars are conductive. This enables to deduce the flux linkage in the rotor slots due to the stator $\mathrm{mmf}$ and the synchronous inductance of each rotor bar by dividing the flux linkage by the stator input current. The induced voltage in each rotor bar is obtained by derivation of the flux linkage. The bar resistance due to skin effect at the slip frequency is then approximated using analytical expressions [12]. Eddy-currents are finally deduced by dividing the induced voltage by the rotor bar impedance. These eddy-currents are called "resistancelimited" because the bar resistance does not depend on the rotor reaction field, which is included afterwards in the air gap flux density. Therefore, the skin effect is the same for the fundamental and for the harmonics of current. This subdomain model is extended in [13] where subdomains equations and EEC equations are solved simultaneously with a transient timestepping method to directly get the rotor reaction field in the air gap. This second method gives accurate results regarding torque ripple and harmonics of induced rotor currents, even if no comparative illustrations are given. The authors say that the computation time for 2500 time steps is less than 5 minutes whereas a linear transient FEA lasts around 105 minutes. However, this SDM requires computing a numerical transient first to reach steady state and the skin effect is still the same for all the space harmonics.

In this paper, a new subdomain model of SCIM is developed for the computation of "skin-limited" eddy-currents in rotor bars by solving Helmholtz's equation and using time-stepping technique to account for rotor motion. Contrary to [9], [10], [11], [13], the problem is solved for each space harmonic of the stator mmf using current sheet approach which enables to model the proper skin effect in the rotor bars. It includes both stator and rotor slotting harmonics in the time and space flux density distribution while only rotor ones are accounted in [8]. Finally the problem is directly solved at steady-state and does not require any numerical transient unlike [10], [13].

The first part presents the modeling assumptions and the physical problem. Then, the Magnetic Vector Potential (MVP) solution and the interface conditions are given for each subdomain. The next section derives the MVP to obtain rotor bar currents, instantaneous torque and electromotive force (EMF). The results are finally validated by a linear transient Finite Element Analysis (FEA) performed on ANSYS Maxwell 2D [14], giving similar results for every electromagnetic quantity.

\section{SUbDOMAIN Model AND Assumptions}

\section{A. General Modeling Assumptions}

The topology of the SCIM subdomain model is illustrated on Fig. 1. In the following, $Z_{s}$ is the number of stator teeth, $Z_{r}$ is the number of rotor teeth and $p$ is the number of pole pairs.

The following assumptions are made:

- The problem is steady-state and solved using timestepping to account for rotor motion for each instant $t$;

- The problem is 2-D: end effects are neglected. The problem is solved using MVP formulation such as $\boldsymbol{A}=$ $A_{z}(r, \theta, t) \mathbf{z}\left(=A_{X}(r, \theta, t) \mathbf{z}\right.$ in each subdomain $\left.X\right)$;

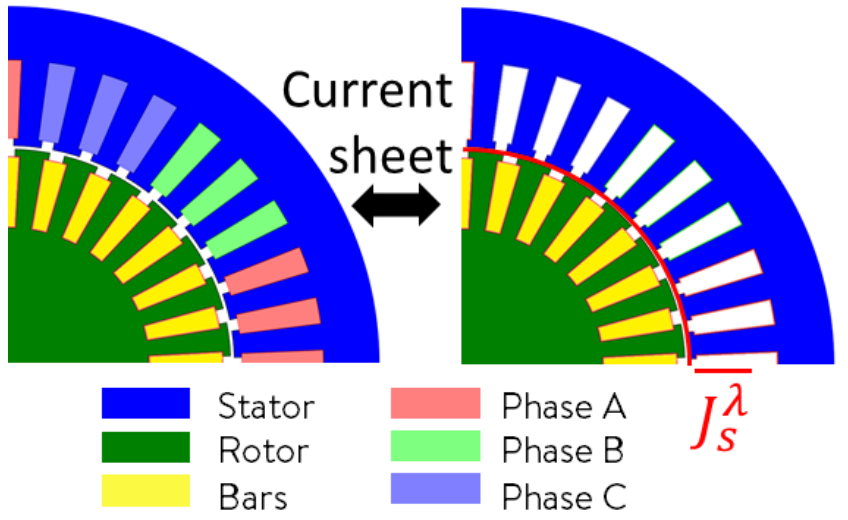

Fig. 1 Subdomain model of an SCIM with current sheet at stator bore accounting for windings in stator slots

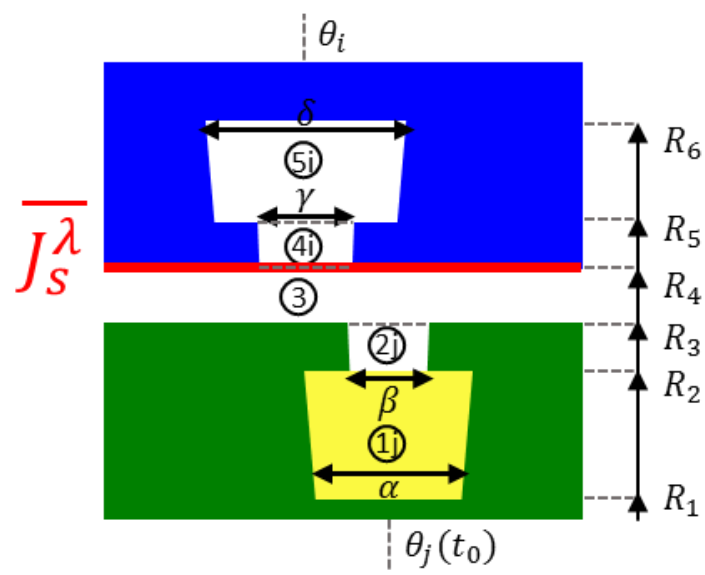

Fig. 2 Subdomain model with geometrical parameters and current sheet

- Geometry is polar: slots and teeth sides are radial segments, slots bottom and teeth top are circular arcs;

- Stator and rotor iron cores are infinitely permeable;

- Rotor angular speed $\Omega$ is constant, with $\Omega=\frac{(1-s) f_{s}}{p}, s$ the fundamental slip and $f_{s}$ the fundamental frequency;

- Rotor bars have a uniform electrical conductivity $\sigma$;

In order to account for the proper skin effect for each space harmonic of the stator mmf, the stator windings are replaced by a superposition of current sheets $\overline{J_{S}^{\lambda}}$ located at the stator inner bore, as shown on Fig. 1 and where $\lambda$ is a specific space harmonic. The expression of $\overline{J_{s}^{\lambda}}$ is given in sub-section II.C. This assumption can be applied to any type of multi-phases windings including double-layer distributed or fractional slot winding.

\section{B. Subdomains Definition}

The subdomain model is presented on Fig. 2. It contains five different types of subdomains:

- $Z_{r}$ rotor slots $(1 j), j \in \llbracket 1, Z_{r} \rrbracket$,

- $Z_{r}$ rotor slot openings $(2 j)$,

- the air gap (3),

- $Z_{s}$ stator slot openings $(4 i), i \in \llbracket 1, Z_{s} \rrbracket$,

- $Z_{s}$ stator slots $(5 i)$.

The geometrical parameters are the bottom radius of the rotor slots $R_{1}$, the top radius of the rotor slots $R_{2}$, the rotor bore radius $R_{3}$, the stator bore radius $R_{4}$, the top radius of the stator slots 
$R_{5}$, the bottom radius of the stator slots $R_{6}$, the rotor slots angular width $\alpha$, the rotor slots opening angular width $\beta$, the stator slots opening angular width $\gamma$ and the stator slots angular width $\delta$. As rotor and stator subdomains are symmetrical with respect to the air gap subdomain, this model is also suited to external rotor topologies.

In the stator reference frame, the angular position $\theta_{i}$ of the $i^{t h}$ stator slots and stator slots opening is:

$$
\theta_{i}=\frac{2 \pi}{Z_{s}}(i-1)+\theta_{i 0}
$$

where $\theta_{i 0}$ is the initial angular position of the first stator slot. The angular position $\theta_{j}$ of the $j^{\text {th }}$ rotor slot and slot opening at the instant $t$ is:

$$
\theta_{j}(t)=\frac{2 \pi}{Z_{r}}(j-1)+\Omega t+\theta_{j 0}
$$

where $\theta_{j 0}$ is the initial angular position of the first rotor slot.

\section{Current Sheet Modeling of Stator Windings}

The stator windings are composed of $N$ conductors per slots and supplied with a balanced three-phase current of magnitude $I_{m}$. The current $I_{i}$ flowing at $t$ in the $i^{t h}$ stator slot is given by:

$$
\bar{I}_{i}(t)=N I_{m} \mathbb{C} *\left[1 \ldots e^{j \frac{(q-1) 2 \pi}{q_{S}}} \ldots e^{j \frac{\left(q_{S}-1\right) 2 \pi}{q_{S}}}\right] * e^{j \omega_{s} t}
$$

where $\mathbb{C}$ is the connection matrix of size $\left[Z_{s}, q_{s}\right]$ which represents the winding distribution of the machine, $q_{s}$ is the number of phases with $q \in \llbracket 2, q_{s} \rrbracket, j=\sqrt{-1}$ and $\omega_{s}=2 \pi f_{s}$. The resulting current sheet distribution $J_{s}(t, \theta)$ in Ampere per meter $(A / m)$ along the stator bore is stepwise. For a distributed winding with integer number of slot per pole and per phase, it can be expressed in Fourier series as:

$$
\begin{aligned}
\overline{J_{s}}(t, \theta) & =\sum_{\lambda} \overline{J_{s}^{\lambda}}(t, \theta) \\
& =\sum_{\lambda} \overline{J_{s, \sin }^{\lambda}}(t) \sin (\lambda p \theta)+\overline{J_{s, \cos }^{\lambda}}(t) \cos (\lambda p \theta)
\end{aligned}
$$

where $\theta$ is the mechanical angle in the stator reference, $\lambda$ is the space harmonic order whose values are:

$$
\lambda=1 \pm 6 \eta \text { where } \eta \in \mathbb{N}
$$

$\overline{J_{S, S i n}^{\lambda}}$ and $\overline{J_{S, \cos }^{\lambda}}$ are the complex magnitude of the $\lambda^{\text {th }}$ component of the current sheet. They are obtained using the classical Fourier expansion:

$$
\begin{aligned}
& \overline{J_{s, \sin }^{\lambda}}(t)=\frac{1}{\pi} \sum_{i=1}^{Z_{s}} \int_{\theta_{i}-\frac{\gamma}{2}}^{\theta_{i}+\frac{\gamma}{2}} \frac{\overline{I_{i}}(t)}{l_{s}} \sin (\lambda p \theta) d \theta \\
& \overline{J_{S, \cos }^{\lambda}}(t)=\frac{1}{\pi} \sum_{i=1}^{Z_{s}} \int_{\theta_{i}-\frac{\gamma}{2}}^{\theta_{i}+\frac{\gamma}{2}} \frac{\overline{I_{i}}(t)}{l_{s}} \cos (\lambda p \theta) d \theta
\end{aligned}
$$

where $l_{s}=\gamma R_{4}$ is the stator slot opening length. Therefore $\frac{I_{i}(t)}{l_{s}}$ is the equivalent linear current density accounting for the $i^{\text {th }}$ slot current. For a fractional slot winding, the values taken by $\lambda$ are given by the "star of slots" method [15]. In case of unbalanced current distribution, $\lambda$ can reach any integer value.

For voltage input, the fundamental of the stator phase current (including magnitude $I_{V}$ and phase $\phi$ ) can be computed using the EEC presented in [9]. Then, the phase current is integrated in this SDM by replacing $I_{m}$ with $I_{V} e^{j \phi}$ in the equation (3) giving $\overline{I_{i}}(t)$. Furthermore, a direct-current (DC) component can be introduced by putting $\omega_{s}=0$ in this same equation (3).

\section{Eddy-currents Modeling in Rotor Bars}

Each space harmonic of the current sheet $\overline{J_{s}^{\lambda}}$ produces either a forward $(\lambda=1,7,13 \ldots)$ or a backward magnetic field $(\lambda=$ $-5,-11,-17 \ldots)$ which induces eddy-current in the conductive rotor bars at the specific pulsation $\omega_{r}^{\lambda}[8]$ :

$$
\omega_{r}^{\lambda}=(1-\lambda(1-s)) \omega_{s}
$$

By considering each single space harmonic of the stator $\mathrm{mmf}$ - meaning a single rotor bar pulsation - at once, the quasi-static Maxwell's equations in the rotor slots subdomains (1j) simplifies into the complex Helmholtz equation [8], [9]:

$$
\frac{\partial^{2} \overline{A_{1 j}^{\lambda}}}{\partial r^{2}}+\frac{1}{r} \frac{\partial \overline{A_{1 j}^{\lambda}}}{\partial r}+\frac{1}{r^{2}} \frac{\partial^{2} \overline{A_{1 j}^{\lambda}}}{\partial \theta^{2}}=j \omega_{r}^{\lambda} \sigma \mu_{0} \mu_{r} \overline{A_{1 j}^{\lambda}}
$$

with $\mu_{r}$ the relative magnetic permeability of the rotor bar. Therefore, the diffusion coefficient $\overline{\alpha_{\lambda}}=\sqrt{j \omega_{r}^{\lambda} \sigma \mu_{0} \mu_{r}}$ accounts for the skin effect which is stronger for higher ranks of space harmonics. Resulting eddy-currents are "skin-limited".

\section{Potential Vector Computation}

\section{A. Introduction}

Except for the rotor slot subdomain for which the complex Helmholtz equation (9) is considered, the complex Laplace equation (10) has to be solved in the other subdomains for every instant $t$ and space harmonics $\lambda$ :

$$
\frac{\partial^{2} \overline{A_{X}^{\lambda}}}{\partial r^{2}}+\frac{1}{r} \frac{\partial \overline{A_{X}^{\lambda}}}{\partial r}+\frac{1}{r^{2}} \frac{\partial^{2} \overline{A_{X}^{\lambda}}}{\partial \theta^{2}}=0
$$

where $X \in\{(2 j) ;(3) ;(4 i) ;(5 i)\}$. In the next part, Equations (9) and (10) are analytically solved by using the separation of variables technique, giving an analytical solution of the MVP in Fourier series for each subdomain with the formulation adopted in [7], [8]. This formulation enables to significantly simplify the expressions of the continuity conditions at the interface between subdomains. It also includes the boundary conditions with the infinitely permeable iron cores. The definition of the iron boundary conditions are not detailed in this article and can be entirely found in [9].

In the next sections, radial and tangential flux densities are computed from the MVP:

$$
\overline{B_{r X}^{\lambda}}(r, \theta, t)=\frac{1}{r} \frac{\partial \overline{A_{X}^{\lambda}}}{\partial \theta} \quad ; \overline{B_{\theta X}^{\lambda}}(r, \theta, t)=-\frac{\partial \overline{A_{X}^{\lambda}}}{\partial r}
$$


Assuming uniform magnetic permeability within subdomains, the magnetic field $\boldsymbol{H}$ is obtained from the flux density $\boldsymbol{B}$ :

$$
\overline{\boldsymbol{B}_{\boldsymbol{X}}^{\lambda}}(r, \theta, t)=\mu_{X} \overline{\boldsymbol{H}_{\boldsymbol{X}}^{\lambda}}(r, \theta, t)
$$

The continuity conditions at an interface between two subdomains concern the MVP and the tangential field such as:

$$
\begin{gathered}
\overline{A_{X}^{\lambda}}-\overline{A_{Y}^{\lambda}}=0 \\
\overline{H_{\theta X}^{\lambda}}-\overline{H_{\theta Y}^{\lambda}}=\overline{J_{s}}
\end{gathered}
$$

where $Y$ is adjacent to $X$ and $\overline{J_{S}}$ is the interface current sheet (in $A / m$ ), here only present at the interface $r=R_{4}$.

\section{B. Notations}

The formulation used in this paper requires the introduction of the following mathematical functions:

$$
\begin{gathered}
P(x, y, z)=\left(\frac{y}{z}\right)^{x}+\left(\frac{y}{z}\right)^{-x} \\
E(x, y, z)=\left(\frac{y}{z}\right)^{x}-\left(\frac{y}{z}\right)^{-x} \\
Q(v, w, x, y, z)=I_{w}(v y) K_{x}(v z)+I_{x}(v z) K_{w}(v y) \\
H(v, w, x, y, z)=I_{w}(v y) K_{x}(v z)-I_{x}(v z) K_{w}(v y)
\end{gathered}
$$

with $I$ and $K$ the modified Bessel functions.

\section{MVP in Rotor Slots Subdomains}

The MVP solution in the rotor slot subdomain is:

$$
\begin{aligned}
& \overline{A_{1 j}^{\lambda}}(r, \theta, t)=-\overline{A_{1 j 0}^{\lambda}} \frac{Q\left(\overline{\alpha_{\lambda}}, 0,1, r, R_{1}\right)}{\overline{\alpha_{\lambda}}} \frac{\left.\overline{H\left(\overline{\alpha_{\lambda}}\right.}, 1,1, R_{1}, R_{2}\right)}{} \\
&+\sum_{m=1}^{\infty} \overline{A_{1 j m}^{\lambda}} \frac{\mathrm{E}_{\sigma}(r)}{\mathrm{E}_{\sigma}^{\prime}\left(R_{2}\right)} \cos \left(\frac{\pi m}{\alpha}\left(\theta-\theta_{j}(t)+\frac{\alpha}{2}\right)\right)
\end{aligned}
$$

where $m$ is a positive integer, $\overline{A_{1 j 0}^{\lambda}}$ and $\overline{A_{1 j m}^{\lambda}}$ are complex integration constants depending on time. They are determined by applying the tangential field continuity with the rotor slot opening:

$$
\begin{aligned}
\overline{A_{1 j 0}^{\lambda}}=-\frac{1}{\alpha} \int_{\theta_{j}(t)-\frac{\beta}{2}}^{\theta_{j}(t)+\frac{\beta}{2}} \overline{H_{\theta 2 j}^{\lambda}}\left(R_{2}, \theta, t\right) d \theta \\
\overline{A_{1 j m}^{\lambda}}=-\frac{2}{\alpha} \int_{\theta_{j}(t)-\frac{\beta}{2}}^{\theta_{j}(t)+\frac{\beta}{2}} \overline{H_{\theta 2 j}^{\lambda}}\left(R_{2}, \theta, t\right) \\
\quad * \cos \left(\frac{\pi m}{\alpha}\left(\theta-\theta_{j}(t)+\frac{\alpha}{2}\right)\right) d \theta
\end{aligned}
$$

$\mathrm{E}_{\sigma}$ and $\mathrm{E}_{\sigma}^{\prime}=\frac{d \mathrm{E}_{\sigma}}{d r}$ depends on $Q$ and $H$ functions such as:

$$
\mathrm{E}_{\sigma}(r)=Q\left(\overline{\alpha_{\lambda}}, \frac{\pi m}{\alpha}-1, \frac{\pi m}{\alpha}, R_{1}, r\right)+Q\left(\overline{\alpha_{\lambda}}, \frac{\pi m}{\alpha}+1, \frac{\pi m}{\alpha}, R_{1}, r\right)
$$

$$
\begin{gathered}
\mathrm{E}_{\sigma}^{\prime}\left(R_{2}\right)=\frac{-2}{\overline{\alpha_{\lambda}}}\left[Q\left(\overline{\alpha_{\lambda}}, \frac{\pi m}{\alpha}-1, \frac{\pi m}{\alpha}, R_{1}, R_{2}\right)+Q\left(\overline{\alpha_{\lambda}}, \frac{\pi m}{\alpha}+1, \frac{\pi m}{\alpha}, R_{1}, R_{2}\right)\right] \\
/\left[H\left(\overline{\alpha_{\lambda}}, \frac{\pi m}{\alpha}-1, \frac{\pi m}{\alpha}-1, R_{1}, R_{2}\right)+H\left(\overline{\alpha_{\lambda}}, \frac{\pi m}{\alpha}-1, \frac{\pi m}{\alpha}+1, R_{1}, R_{2}\right)\right. \\
\left.+H\left(\overline{\alpha_{\lambda}}, \frac{\pi m}{\alpha}+1, \frac{\pi m}{\alpha}-1, R_{1}, R_{2}\right)+H\left(\overline{\alpha_{\lambda}}, \frac{\pi m}{\alpha}+1, \frac{\pi m}{\alpha}+1, R_{1}, R_{2}\right)\right]
\end{gathered}
$$

\section{MVP in Rotor Slot Openings Subdomains}

The MVP solution in the rotor slot opening subdomain is:

$$
\begin{array}{r}
\overline{A_{2 j}^{\lambda}}(r, \theta, t)=\overline{A_{2 j 0}^{\lambda}}+\overline{B_{2 j 0}^{\lambda}} \ln (r) \\
+\sum_{k=1}^{\infty}\left[\overline{A_{2 j k}^{\lambda}} \frac{E\left(\frac{\pi k}{\beta}, r, R_{3}\right)}{E\left(\frac{\pi k}{\beta}, R_{2}, R_{3}\right)}+\overline{B_{2 j k}^{\lambda}} \frac{E\left(\frac{\pi k}{\beta}, R_{2}, r\right)}{E\left(\frac{\pi k}{\beta}, R_{2}, R_{3}\right)}\right] \\
* \cos \left(\frac{\pi k}{\beta}\left(\theta-\theta_{j}(t)+\frac{\beta}{2}\right)\right)
\end{array}
$$

where $k$ is a positive integer, $\overline{A_{2 j 0}^{\lambda}}, \overline{B_{2 j 0}^{\lambda}}, \overline{A_{2 j k}^{\lambda}}$, and $\overline{B_{2 j k}^{\lambda}}$ are complex integration constants depending on time. They are determined by applying the potential continuity with the adjacent rotor slot subdomain:

$$
\begin{array}{r}
\overline{A_{2 j 0}^{\lambda}}+\overline{B_{2 j 0}^{\lambda}} \ln \left(R_{2}\right)=\frac{1}{\beta} \int_{\theta_{j}(t)-\frac{\beta}{2}}^{\theta_{j}(t)+\frac{\beta}{2} \overline{A_{1 j}^{\lambda}}}\left(R_{2}, \theta, t\right) d \theta \\
\overline{A_{2 j k}^{\lambda}}=\frac{2}{\beta} \int_{\theta_{j}(t)-\frac{\beta}{2}}^{\theta_{j}(t)+\frac{\beta}{2} \overline{A_{1 j}^{\lambda}}}\left(R_{2}, \theta, t\right) \\
\quad * \cos \left(\frac{\pi k}{\beta}\left(\theta-\theta_{j}(t)+\frac{\beta}{2}\right)\right) d \theta
\end{array}
$$

and with the air gap subdomain:

$$
\begin{aligned}
& \overline{A_{2 j 0}^{\lambda}}+\overline{B_{2 j 0}^{\lambda}} \ln \left(R_{3}\right)=\frac{1}{\beta} \int_{\theta_{j}(t)-\frac{\beta}{2}}^{\theta_{j}(t)+\frac{\beta}{2}} \overline{A_{3}^{\lambda}}\left(R_{3}, \theta, t\right) d \theta \\
& \overline{B_{2 j k}^{\lambda}}=\frac{2}{\beta} \int_{\theta_{j}(t)-\frac{\beta}{2}}^{\theta_{j}(t)+\frac{\beta}{2}} \overline{A_{3}^{\lambda}}\left(R_{3}, \theta, t\right) \\
& * \cos \left(\frac{\pi k}{\beta}\left(\theta-\theta_{j}(t)+\frac{\beta}{2}\right)\right) d \theta
\end{aligned}
$$

\section{E. MVP in the Air Gap Subdomain}

The MVP solution in the air gap subdomain is:

$$
\begin{aligned}
& \overline{A_{3}^{\lambda}}(r, \theta, t)=\overline{A_{30}^{\lambda}}+\overline{B_{30}^{\lambda}} \ln (r)+ \\
& \sum_{n=1}^{\infty}\left[\overline{A_{3 n}^{\lambda}} \frac{R_{3} P\left(n, r, R_{4}\right)}{n E\left(n, R_{3}, R_{4}\right)}-\overline{B_{3 n}^{\lambda}} \frac{R_{4} P\left(n, R_{3}, r\right)}{n E\left(n, R_{3}, R_{4}\right)}\right] \cos (n \theta) \\
& +\left[\overline{C_{3 n}^{\lambda}} \frac{R_{3} P\left(n, r, R_{4}\right)}{n E\left(n, R_{3}, R_{4}\right)}-\overline{D_{3 n}^{\lambda}} \frac{R_{4} P\left(n, R_{3}, r\right)}{n E\left(n, R_{3}, R_{4}\right)}\right] \sin (n \theta)
\end{aligned}
$$

where $n$ is a positive integer, $\overline{A_{30}^{\lambda}}, \overline{B_{30}^{\lambda}}, \overline{A_{3 n}^{\lambda}}, \overline{B_{3 n}^{\lambda}}, \overline{C_{3 n}^{\lambda}}$ and $\overline{D_{3 n}^{\lambda}}$ are complex integration constants depending on time. They are 
determined by applying the tangential field continuity with all rotor slot opening subdomains:

$$
\begin{aligned}
& \overline{B_{30}^{\lambda}}=-\frac{1}{2 \pi} \sum_{j=1}^{Z_{r}} \int_{\theta_{j}(t)-\frac{\beta}{2}}^{\theta_{j}(t)+\frac{\beta}{2}} \overline{H_{\theta 2 j}^{\lambda}}\left(R_{3}, \theta, t\right) d \theta \\
& \overline{A_{3 n}^{\lambda}}=-\frac{1}{\pi} \sum_{j=1}^{Z_{r}} \int_{\theta_{j}(t)-\frac{\beta}{2}}^{\theta_{j}(t)+\frac{\beta}{2}} \overline{H_{\theta 2 j}^{\lambda}}\left(R_{3}, \theta, t\right) \cos (n \theta) d \theta \\
& \overline{C_{3 n}^{\lambda}}=-\frac{1}{\pi} \sum_{j=1}^{Z_{r}} \int_{\theta_{j}(t)-\frac{\beta}{2}}^{\theta_{j}(t)+\frac{\beta}{2}} \overline{H_{\theta 2 j}^{\lambda}}\left(R_{3}, \theta, t\right) \sin (n \theta) d \theta
\end{aligned}
$$

and with all stator slot opening subdomains:

$$
\begin{aligned}
& \overline{B_{30}^{\lambda}}=-\frac{1}{2 \pi} \sum_{i=1}^{Z_{s}} \int_{\theta_{i}-\frac{\delta}{2}}^{\theta_{i}+\frac{\delta}{2}} \overline{H_{\theta 4 i}^{\lambda}}\left(R_{4}, \theta, t\right) d \theta \\
& \overline{B_{3 n}^{\lambda}}=\overline{J_{s, \cos }^{n}}(t)-\frac{1}{\pi} \sum_{i=1}^{Z_{s}} \int_{\theta_{i}-\frac{\delta}{2}}^{\theta_{i}+\frac{\delta}{2}} \overline{H_{\theta 4 i}^{\lambda}}\left(R_{4}, \theta, t\right) \cos (n \theta) d \theta \\
& \overline{D_{3 n}^{\lambda}}=\overline{J_{s, s i n}^{n}}(t)-\frac{1}{\pi} \sum_{i=1}^{Z_{s}} \int_{\theta_{i}-\frac{\delta}{2}}^{\theta_{i}+\frac{\delta}{2}} \overline{H_{\theta 4 i}^{\lambda}}\left(R_{4}, \theta, t\right) \sin (n \theta) d \theta
\end{aligned}
$$

where:

$$
\begin{array}{ll}
\overline{J_{s, \cos }^{n}}(t)=\overline{J_{s, \cos }^{\lambda}}(t) \quad \text { for } n=|\lambda p| \\
\overline{J_{s, \sin }^{n}}(t)=\overline{J_{s, \text { sin }}^{\lambda}}(t) & \text { for } n=|\lambda p| \\
\overline{J_{s, \cos }^{n}}(t)=\overline{J_{s, \sin }^{n}}(t)=0 & \text { else }
\end{array}
$$

\section{F. MVP in Stator Slot Openings Subdomains}

As for the rotor slot openings, the MVP solution in the stator slot subdomain is:

$$
\begin{array}{r}
\overline{A_{4 i}^{\lambda}}(r, \theta, t)=\overline{A_{4 i 0}^{\lambda}}+\overline{B_{4 i 0}^{\lambda}} \ln (r) \\
+\sum_{v=1}^{\infty}\left[\overline{A_{4 i v}^{\lambda}} \frac{E\left(\frac{\pi v}{\delta}, r, R_{5}\right)}{E\left(\frac{\pi v}{\delta}, R_{4}, R_{5}\right)}+\overline{B_{4 i v}^{\lambda}} \frac{E\left(\frac{\pi v}{\delta}, R_{4}, r\right)}{E\left(\frac{\pi v}{\delta}, R_{4}, R_{5}\right)}\right] \\
* \cos \left(\frac{\pi v}{\delta}\left(\theta-\theta_{i}+\frac{\delta}{2}\right)\right)
\end{array}
$$

where $v$ is a positive integer, $\overline{A_{4 i 0}^{\lambda}}, \overline{B_{4 i 0}^{\lambda}}, \overline{A_{4 i v}^{\lambda}}$ and $\overline{B_{4 i v}^{\lambda}}$ are complex integration constants depending on time. They are determined by applying the potential continuity with the air gap subdomain:

$$
\overline{A_{4 i 0}^{\lambda}}+\overline{B_{4 i 0}^{\lambda}} \ln \left(R_{4}\right)=\frac{1}{\delta} \int_{\theta_{i}-\frac{\delta}{2}}^{\theta_{i}+\frac{\delta}{2}} \overline{A_{3}^{\lambda}}\left(R_{4}, \theta, t\right) d \theta
$$

$$
\overline{A_{4 i v}^{\lambda}}=\frac{2}{\delta} \int_{\theta_{i}-\frac{\delta}{2}}^{\theta_{i}+\frac{\delta}{2}} \overline{A_{3}^{\lambda}}\left(R_{4}, \theta, t\right) \cos \left(\frac{\pi v}{\delta}\left(\theta-\theta_{i}+\frac{\delta}{2}\right)\right) d \theta
$$

and with the adjacent stator slot subdomain:

$$
\begin{aligned}
& \overline{A_{4 i 0}^{\lambda}}+\overline{B_{4 i 0}^{\lambda}} \ln \left(R_{5}\right)=\frac{1}{\delta} \int_{\theta_{i}-\frac{\delta}{2}}^{\theta_{i}+\frac{\delta}{2}} \overline{A_{5 i}^{\lambda}}\left(R_{5}, \theta, t\right) d \theta \\
& \overline{B_{4 i v}^{\lambda}}=\frac{2}{\delta} \int_{\theta_{i}-\frac{\delta}{2}}^{\theta_{i}+\frac{\delta}{2}} \overline{A_{5 i}^{\lambda}}\left(R_{5}, \theta, t\right) \cos \left(\frac{\pi v}{\delta}\left(\theta-\theta_{i}+\frac{\delta}{2}\right)\right) d \theta
\end{aligned}
$$

\section{G. MVP in Stator Slots Subdomains}

The MVP solution in the stator slot subdomain is:

$$
\begin{aligned}
& \overline{A_{5 i}^{\lambda}}(r, \theta, t)=\overline{A_{5 i 0}^{\lambda}} \\
& +\sum_{l=1}^{\infty} \overline{A_{5 i l}^{\lambda}} \frac{R_{5} \gamma P\left(\frac{\pi l}{\gamma}, r, R_{6}\right)}{\pi l E\left(\frac{\pi l}{\gamma}, R_{5}, R_{6}\right)} \cos \left(\frac{\pi l}{\gamma}\left(\theta-\theta_{i}+\frac{\gamma}{2}\right)\right)
\end{aligned}
$$

where $l$ is a positive integer, $\overline{A_{5 i 0}^{\lambda}}$ and $\overline{A_{5 i l}^{\lambda}}$ are complex integration constants depending on time. They are determined by applying the tangential field continuity with the adjacent stator slot opening subdomain:

$$
\begin{aligned}
& -\frac{d \overline{A_{5 i 0}^{\lambda}}}{d r}=0=-\frac{1}{\gamma} \int_{\theta_{i}-\frac{\delta}{2}}^{\theta_{i}+\frac{\delta}{2}} \overline{H_{\theta 4 i}^{\lambda}}\left(R_{5}, \theta, t\right) d \theta \\
& \overline{A_{5 i l}^{\lambda}}=-\frac{2}{\gamma} \int_{\theta_{i}-\frac{\delta}{2}}^{\theta_{i}+\frac{\delta}{2}} \overline{H_{\theta 4 i}^{\lambda}}\left(R_{5}, \theta, t\right) \cos \left(\frac{\pi l}{\gamma}\left(\theta-\theta_{i}+\frac{\gamma}{2}\right)\right) d \theta
\end{aligned}
$$

\section{H. Matrix Resolution}

In the resolution process, the time dependency concerns:

- the continuity equations between air gap (3) and rotor slot openings $(2 j)$, which account for rotor motion;

- the continuity equations between air gap (3) and stator slot openings ( $4 i$ ) which include the rotation of the current sheet $\overline{J_{S}^{\lambda}}(t, \theta)$.

For the numerical resolution, only a finite number $N_{\lambda}$ of space harmonics is considered. The Fourier series of each MVP solution is truncated as well to a finite number of harmonics $\{M, K, N, V, L\}$ corresponding to the integers $\{m, k, n, v, l\}$. Therefore, the total number of equations $N_{e q}$ is equal to the number of integration constants such as $N_{e q}=(M+2 K+$ 3) $Z_{r}+4 N+2+(2 V+L+3) Z_{s}$. However, once the continuity equations are developed, some of the integration constants are equal to zero. Moreover, the number of equations can be reduced by introducing the periodicity coefficient $c=$ $\operatorname{gcd}\left(p, Z_{r}, Z_{s}\right)$ and only accounting for $Z_{r} / c$ rotor subdomains and $Z_{s} / c$ stator subdomains [6]. The resulting set of equations is written in matrix form: 


$$
\mathbb{M}(\lambda, t) \times \boldsymbol{C}(\lambda, t)=\boldsymbol{S}(t)
$$

where $\mathbf{M}$ is the topological matrix of size $N_{e q}{ }^{2}, \boldsymbol{C}$ is the vector of integration constants and $\boldsymbol{S}$ is the source vector including the current sheet. The total number of system resolutions (45) is $N_{\lambda} * N_{\Delta t}$, where $N_{\Delta t}$ is the number of time steps and $\Delta t$ is the time step value.

For every instant $t$, the total MVP solution is obtained by summing the contribution of each $\lambda$ by mean of superposition principle. For example, the total air gap integration constants are obtained with the following equations:

$$
X_{3 n}=\Re\left[\sum_{\lambda} \overline{X_{3 n}^{\lambda}}\right] \text { with } X \in\{A, B, C, D\}
$$

\section{ELECTROMAGNETIC QUANTITIES COMPUTATION}

\section{A. Current in the $j^{\text {th }}$ rotor bar}

The current density in the $\mathrm{j}^{\text {th }}$ rotor bar at $t$ is given by:

$$
\overline{J_{j}}(r, \theta, t)=\sum_{\lambda}-j \omega_{r}^{\lambda} \sigma \overline{A_{1 j}^{\lambda}}(r, \theta, t)
$$

The total current $I_{j}$ flowing in the $\mathrm{j}^{\text {th }}$ rotor bar is therefore obtained by integrating the current density over the bar surface:

$$
I_{j}(t)=\Re\left[\int_{\theta_{j}(t)-\frac{\alpha}{2}}^{\theta_{j}(t)+\frac{\alpha}{2}} \int_{R_{1}}^{R_{2}} \overline{J_{j}}(r, \theta, t) r d \theta d r\right]
$$

where $\mathfrak{R}[$.$] is the real part operator. Considering equations (19)$ and (47), knowing that $\overline{\alpha_{\lambda}}=\sqrt{\iota \omega_{r}^{\lambda} \sigma \mu_{0} \mu_{r}}$ and noticing that:

$$
\int_{R_{1}}^{R_{2}} r Q\left(\overline{\alpha_{\lambda}}, 0,1, r, R_{1}\right) d r=-\frac{R_{2}}{\overline{\alpha_{\lambda}}} H\left(\overline{\alpha_{\lambda}}, 1,1, R_{1}, R_{2}\right)
$$

the current $I_{j}$ in the $\mathrm{j}^{\text {th }}$ rotor bar can be simply expressed as:

$$
I_{j}(t)=-\frac{\alpha R_{2}}{\mu_{0} \mu_{r}} \Re\left[\sum_{\lambda} \overline{A_{1 j 0}^{\lambda}}\right]
$$

\section{B. Instantaneous torque}

The instantaneous torque $T(t)$ is computed by integrating the tangential component of the Maxwell stress tensor along a circular path inside the air gap subdomain:

$$
T(t)=\frac{L R_{g}{ }^{2}}{\mu_{0}} \sum_{\lambda} \int_{0}^{2 \pi} \frac{1}{2} \mathfrak{R}\left[\left(\overline{B_{r 3}^{\lambda}}{\overline{B_{\theta 3}^{\lambda}}}^{*}\right)\left(R_{g}, \theta, t\right)\right] d \theta
$$

where $L$ is the axial length of the machine, $R_{g}$ is a radius between $R_{3}$ and $R_{4}$ and [.] ${ }^{*}$ is the complex conjugate operator.

\section{Electromotive force}

The electromotive force $\boldsymbol{E}$ is obtained by derivating the flux linkage $\boldsymbol{\varphi}$ within each stator coil:

$$
\boldsymbol{E}(t)=N \mathbb{C}^{T} \frac{\boldsymbol{\varphi}(t+\Delta t)-\boldsymbol{\varphi}(t)}{\Delta t}
$$

where $\boldsymbol{\varphi}=\left[\varphi_{1} \ldots \varphi_{i} \ldots \varphi_{Z_{s}}\right]$. Each $\varphi_{i}$ is computed by integrating the potential over the $i^{\text {th }}$ stator slot surface:

$$
\varphi_{i}(t)=\frac{L}{S_{i}} \int_{\theta_{i}-\frac{\gamma}{2}}^{\theta_{i}+\frac{\gamma}{2}} \int_{R_{5}}^{R_{6}} A_{5 i}(r, \theta, t) r d \theta d r
$$

where $S_{i}=\frac{\gamma}{2}\left(R_{6}{ }^{2}-R_{5}{ }^{2}\right)$ is the stator slot surface.

However, the potential $A_{5 i}(r, \theta, t)$ used in equation (53) must be different from the computed stator slot potential $\overline{A_{5 i}^{\lambda}}(r, \theta, t)$ (defined in equation (42)) because this latter is due to the virtual current sheet $\overline{J_{S}^{\lambda}}(t, \theta)$ and not to the actual current density in the stator slots. The stator slot potential $A_{5 i}(r, \theta, t)$ accounting for the actual current density in stator slots can be reconstructed by solving an additional smaller subdomain problem. This latter represents only the stator subdomains (slots and slot openings) whose boundary conditions with the air gap are given thanks to the knowledge of the air gap integration constants previously computed (from the matrix resolution in the Section III.H).

The expression of the MVP in the stator slot opening is the same as in equation (37) but without the $\lambda$-dependency. The analytical solution of the stator slot MVP with inner current density is given by [7]:

$$
\begin{aligned}
& A_{5 i}(r, \theta, t)=A_{5 i 0}+\left(R_{6}{ }^{2} \ln (r)-\frac{r^{2}}{2}\right) \mu_{0} J_{i}(t) \\
& +\sum_{l=1}^{L} A_{5 i l} \frac{R_{5} \gamma P\left(\frac{\pi l}{\gamma}, r, R_{6}\right)}{\pi l E\left(\frac{\pi l}{\gamma}, R_{5}, R_{6}\right)} \cos \left(\frac{\pi l}{\gamma}\left(\theta-\theta_{i}+\frac{\gamma}{2}\right)\right)
\end{aligned}
$$

where $l$ is a positive integer, $J_{i}(t)$ is the current density in the $i^{\text {th }}$ stator slot given by:

$$
J_{i}(t)=\Re\left[\frac{\overline{I_{i}}(t)}{S_{i}}\right]
$$

and $A_{5 i 0}$ and $A_{5 i l}$ are real integration constants depending on time. $A_{5 i 0}$ is determined thanks to the new tangential field continuity (replacing equation (43)) with the stator slot opening:

$$
\left(R_{5}-\frac{R_{6}{ }^{2}}{R_{5}}\right) J_{i}(t)=-\frac{1}{\gamma} \int_{\theta_{i}-\frac{\delta}{2}}^{\theta_{i}+\frac{\delta}{2}} H_{\theta 4 i}\left(R_{5}, \theta, t\right) d \theta
$$

Hence, the new set of six integration constants in stator slot openings and stator slots $\left\{A_{4 i 0}, B_{4 i 0}, A_{4 i v}, B_{4 i v}, A_{5 i 0}, B_{5 i l}\right\}$ is obtained by solving again the six continuity equations $\{(38)$, (39), (40), (41), (56), (44)\}. Here, the system resolution is simultaneously done for all time steps because the topological matrix does not depend on time. The size of the topological matrix is $\left[(2 V+L+3) Z_{s}\right]^{2}$ and the size of the source vector is $(2 V+L+3) Z_{s} \times N_{\Delta t}$.

By substituting (54) in (53), flux linkage $\varphi_{i}$ becomes:

$$
\begin{aligned}
\varphi_{i}(t) & =L A_{5 i 0}+\frac{\gamma L R_{6}^{4} \mu_{0} J_{i}(t)}{16 S_{i}} \\
& *\left[\left(\frac{R_{5}}{R_{6}}\right)^{4}+\left(\frac{R_{5}}{R_{6}}\right)^{2}\left(2-4 \ln \left(R_{5}\right)\right)+4 \ln \left(R_{6}\right)-3\right]
\end{aligned}
$$


TABLE I LIST OF GEOMETRICAL PARAMETERS

\begin{tabular}{|l|c|c|}
\hline Machine parameters & Symbol & Value [Unit] \\
\hline Nominal supply frequency & $f_{s}$ & $50[\mathrm{~Hz}]$ \\
\hline Stator phase current magnitude & $I_{m}$ & $20[\mathrm{~A}]$ \\
\hline Number of turns per slot & $N$ & 15 \\
\hline Number of rotor bars & $Z_{r}$ & 28 \\
\hline Number of stator slots & $Z_{s}$ & 36 \\
\hline Number of pole pairs & $p$ & 2 \\
\hline Axial length & $L$ & $200[\mathrm{~mm}]$ \\
\hline Rotor slot bottom radius & $R_{1}$ & $38[\mathrm{~mm}]$ \\
\hline Rotor slot top radius & $R_{2}$ & $58[\mathrm{~mm}]$ \\
\hline Rotor bore radius & $R_{3}$ & $60[\mathrm{~mm}]$ \\
\hline Stator bore radius & $R_{4}$ & $61[\mathrm{~mm}]$ \\
\hline Stator slot top radius & $R_{5}$ & $63[\mathrm{~mm}]$ \\
\hline Stator slot bottom radius & $R_{6}$ & $85[\mathrm{~mm}]$ \\
\hline Rotor slot angular width & $\alpha$ & $0.1122[\mathrm{rad}]$ \\
\hline Rotor slot opening angular width & $\beta$ & $0.0674[\mathrm{rad}]$ \\
\hline Stator slot opening angular width & $\delta$ & $0.0524[\mathrm{rad}]$ \\
\hline Stator slot angular width & $\gamma$ & $0.0873[\mathrm{rad}]$ \\
\hline Rotor bar electrical conductivity & $\sigma$ & $58 e 6[\mathrm{~S}]$ \\
\hline Rotor bar relative permeability & $\mu_{r}$ & 1 \\
\hline
\end{tabular}

\section{COMPARISON AND VALIDATION WITH FEA}

\section{A. Study Cases}

\section{1) Topology of the Studied Machine}

The studied machine is the same as in [9]: it is a SCIM with 2 poles pairs, 36 stator teeth and 28 rotor bars. The parameters are given in TABLE I. The stator winding is a single layer distributed winding whose connection matrix $\mathbb{C}$ for one pole is:

$$
\mathbb{C}=\left[\begin{array}{ccccccccc}
1 & 1 & 1 & 0 & 0 & 0 & 0 & 0 & 0 \\
0 & 0 & 0 & 0 & 0 & 0 & 1 & 1 & 1 \\
0 & 0 & 0 & -1 & -1 & -1 & 0 & 0 & 0
\end{array}\right]
$$

\section{2) Choice of Harmonic Numbers}

For both cases, the number of harmonics in each subdomain is chosen as:

- $\quad N=400>10 Z_{s}$ in the air gap, to account for at least 10 ranks of stator and rotor slotting harmonics;

- $M=V=2$ in the rotor slots and stator slot openings;

- $K=L=4$ in rotor slot openings and stator slots.

The number of harmonics in the slots subdomains are very small because only the first space harmonics have an impact on the radial and tangential flux densities. However, for a better estimation of the flux density inside the slots, $K, V \& L$ can be increased up to 10 , whereas $M$ must be inferior to 5 so the Bessel functions can be numerically estimated.

Due to periodicity, the number of rotor and stator subdomains are divided by $\operatorname{gcd}\left(p, Z_{r}, Z_{s}\right)=2$. Moreover, odd harmonics of rank $n$ in the air gap are null. Consequently, a time step takes less than $50 \mathrm{~ms}$ of computation time (less than $25 \mathrm{~ms}$ if no induced currents are computed because the numerical system is real), multiplied by the number of space harmonics $N_{\lambda}$.

Therefore, it can be very time-consuming to account for the right diffusion coefficient associated to each space harmonic. It is proposed to only consider the first $N_{\lambda}$ harmonics of the stator mmf independently as "skin-limited" (for $\eta \leq N_{\lambda}$ in equation (5)) while the higher space harmonics (for $\eta>N_{\lambda}$ ) are considered as "resistance-limited" with the same diffusion coefficient $\overline{\alpha_{\lambda}}=\overline{\alpha_{\left|1 \pm 6 N_{\lambda}\right|}}$ as the last "skin-limited" space harmonic (for $\eta=N_{\lambda}$ ). These higher space harmonics (for $\eta>$ $N_{\lambda}$ ) are all included in the current sheet of the last "skin-limited" harmonic by modifying equation (36) such as:

$$
\begin{aligned}
& \overline{J_{s, \text { cos }}^{n}}(t)=\overline{J_{s, \text { cos }}^{\lambda}}(t) \text { for } n \in\left\{\left|1 \pm 6 N_{\lambda}\right| p \leq|\lambda p| \leq N\right\} \\
& \overline{J_{s, \text { sin }}^{n}}(t)=\overline{J_{s, \text { sin }}^{\lambda}}(t) \text { for } n \in\left\{\left|1 \pm 6 N_{\lambda}\right| p \leq|\lambda p| \leq N\right\} \\
& \overline{J_{s, \text { cos }}^{n}}(t)=\overline{J_{s, \text { sin }}^{n}}(t)=0 \mathrm{else}
\end{aligned}
$$

Therefore, the eddy-currents induced by the higher space harmonics have the proper frequency $\omega_{r}^{\lambda}$ but their skin effect is underestimated because the diffusion coefficient is $\overline{\alpha_{\lambda}}=$ $\overline{\alpha_{\left|1 \pm 6 N_{\lambda}\right|}}$ for $>N_{\lambda}$. The error generated by this simplification is insignificant if $N_{\lambda}$ is chosen such as the skin effect is already very strong for $\overline{\alpha_{\left|1 \pm 6 N_{\lambda}\right|}}$ and so the model is quasi "skin-limited". For this particular machine, $N_{\lambda}=5$ is a good compromise between accuracy and computation time. If $N_{\lambda}=1$ is chosen, the model reduces to the classical "resistance-limited" approach used in previous works, as the skin effect and so the rotor bar resistance are the same for the fundamental and for every space harmonics.

\section{3) Simulation setup}

The machine is simulated at both no-load and heavy load condition. At no-load state, slip is strictly null as if the rotor would be driven by an external machine at synchronous speed. The simulation duration is equal to a rotor mechanical period $p /\left((1-s) f_{s}\right)=0.04 s$ and time step value is $\Delta t=0.0001 s$. Under heavy load condition, slip is equal to $10 \%$ which is quite high in reality but this value enables to reduce the FEA computation time and limit spectral leakage. The simulation duration is equal to a rotor electrical period $1 /\left(s f_{s}\right)=0.2 s$ and time step value is still $\Delta t=0.0001 \mathrm{~s}$. In both cases, the time step value is very small in order to catch high frequencies in the flux density and to compute accurately the magnetic forces.

In the following, the subdomain model is validated by Maxwell 2D transient simulations with sinusoidal input phase current. Due to periodicity and antisymmetry, only a fourth of the machine is modelled. Most of the computation time is dedicated to achieve the numerical transient and reach steady state, which amounts to around 1200 time steps $(0.12 s)$ at noload case and 28000 time steps $(2.8 s)$ for the load case. The computation time per time step is around $6 s$ during the numerical transient and is a bit longer when recording the steady state due to data storage.

The overall computation times are compared in TABLE II. The model shows very good performances as it reduces the computation time up to a factor 350 for the load case between Maxwell 2D (including transient) and $\operatorname{SDM}\left(N_{\lambda}=5\right)$.

TABLE II COMPUTATION TIME COMPARISON

\begin{tabular}{|c|c|c|c|c|c|c|}
\hline \multirow{2}{*}{ Method } & \multicolumn{2}{|c|}{ Transient } & \multicolumn{2}{c|}{ Steady State } & \multicolumn{2}{c|}{ Total } \\
\cline { 2 - 7 } & NL & Load & NL & Load & NL & Load \\
\hline Maxwell 2D & $2 h$ & $46 h$ & $40 m$ & $3 h 20$ & $2 h 40 m$ & $49 h$ \\
\hline SDM $\left(N_{\lambda}=5\right)$ & - & - & $1 m 30 s$ & $8 m 20 s$ & $1 m 30 s$ & $8 m 20 s$ \\
\hline $\operatorname{SDM}\left(N_{\lambda}=1\right)$ & - & - & $10 s$ & $1 m 40 s$ & $10 s$ & $1 m 40 s$ \\
\hline
\end{tabular}



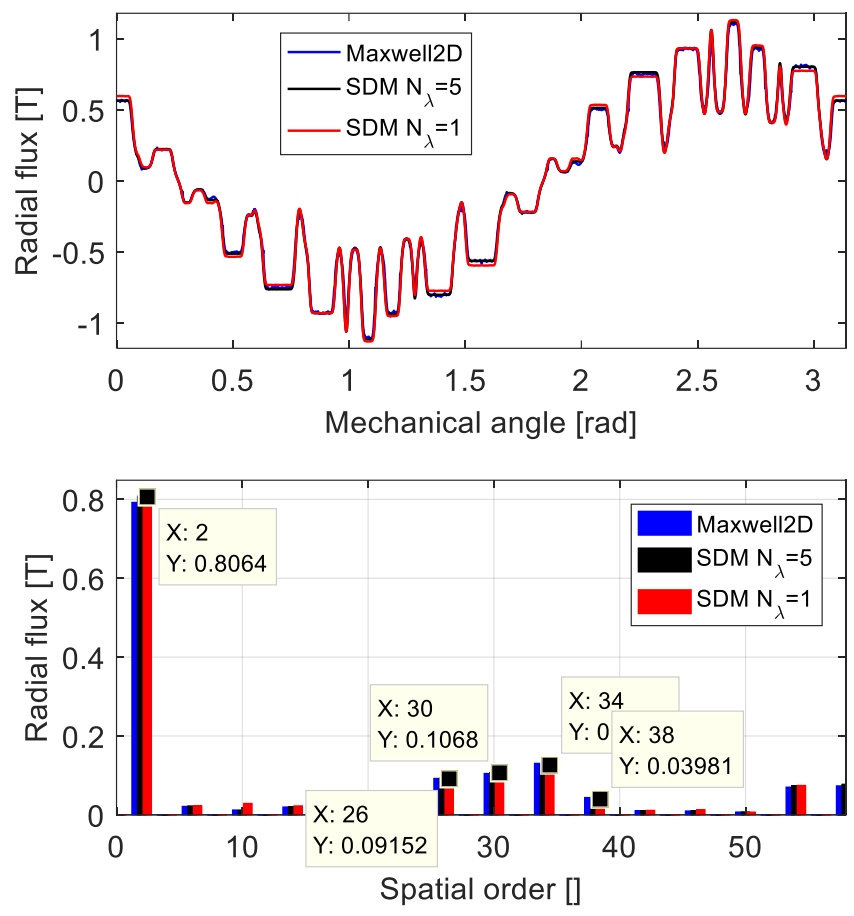

Fig. 3 Radial flux density over space ( $\left(1^{\text {st }}\right.$ time step) at no-load and FFT.

For SDM, the computation time required to compute the electromagnetic quantities from the integration constants is negligible compared with the resolution time in itself (only a few seconds).

\section{B. Results at No-load Case $(s=0)$}

\section{1) Radial and tangential air gap flux densities}

The resulting radial and tangential flux densities at no-load are shown on Fig. 3 to Fig. 5. The developed SDM (with $N_{\lambda}=$ 5 ) is in great accordance with the linear FEA while the "resistance-limited" SDM shows slight discrepancies due to the fact that rotor $\mathrm{mmf}$ is neglected.

For the time plot on Fig. 4, only a fourth of the rotor mechanical period is represented. At no-load, the radial component is much greater than the tangential one. The highest peak in the flux density FFT is naturally the fundamental of the radial flux density at $(f, r)=(50 \mathrm{~Hz}, 2)=\left(f_{s}, p\right)$, where $r$ is the spatial frequency (also called wavenumber) and $f$ is the time frequency. The spatial harmonics at $r=\{34 ; 38\}=Z_{s} \pm p$ are due to the stator mmf stepwise distribution and slotting effect. Spatial harmonics at $r=\{26 ; 30\}=Z_{r} \pm p$ are mainly due to rotor slotting as the rotor $\mathrm{mmf}$ is very small at no-load.

Besides, rotor slotting induces harmonics in the flux density time spectrum, named Principal Slotting Harmonics (PSH) (also visible in stator currents) at the following frequencies [16]:

$$
f_{p s h}^{k}=\left(k \frac{Z_{r}}{p}(1-s) \pm 1\right) f_{s} ; \quad k=1,2,3 \ldots
$$

The first rotor slotting harmonics are for $k=1$, at $f_{p s h}^{1}=$ $\{650 \mathrm{~Hz} ; 750 \mathrm{~Hz}\}$ as shown on Fig. 4.

2) Bar currents, torque and Electromotive Force (EMF)

Rotor bar currents at no-load are shown on Fig. 6 and Fig. 7. They are induced at the frequencies given by equation (8) in the
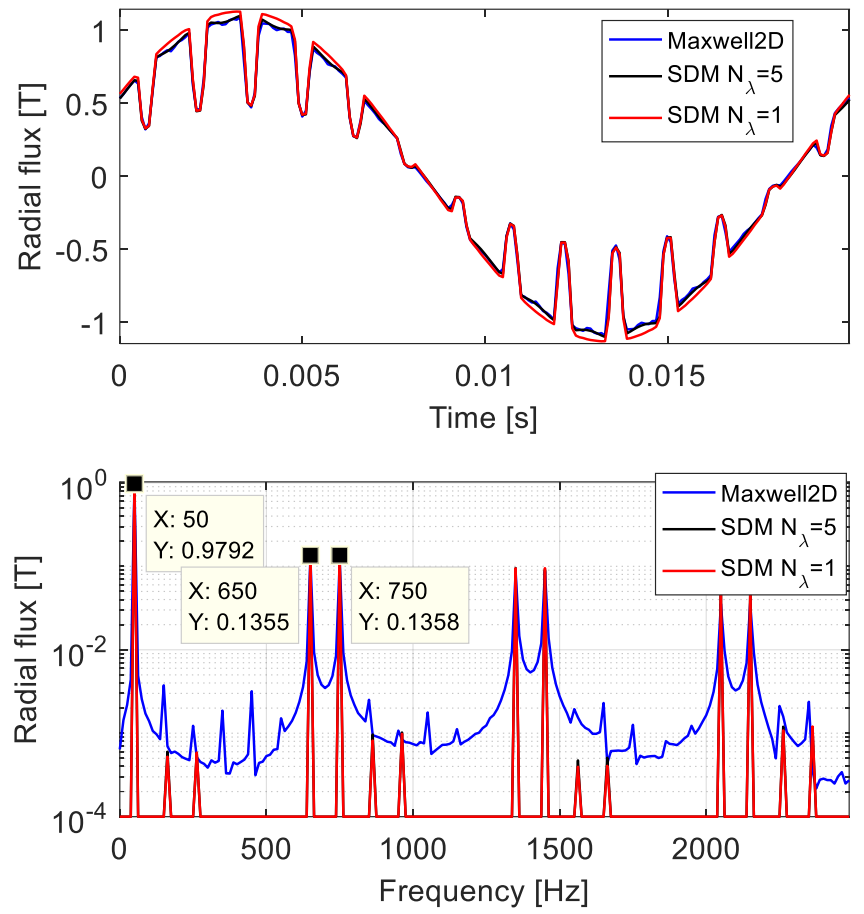

Fig. 4 Radial flux density over time at $\theta=0 \mathrm{rad}$ at no-load and FFT.

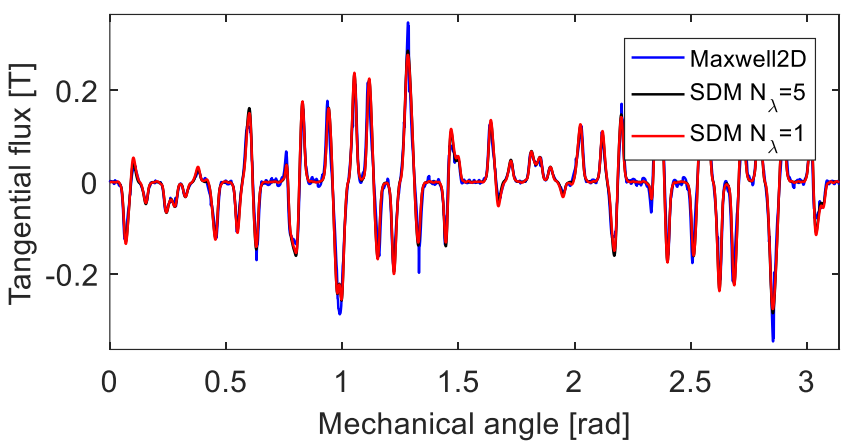

Fig. 5 Tangential flux density over space ( $1^{\text {st }}$ time step) at no-load.

rotor referential. Results given by the "skin-limited" approach $\left(N_{\lambda}=5\right)$ are very close to the FEA. The fundamental of the rotor current is null because the fundamental of the stator mmf and the rotor are synchronous. The first rotor current harmonics are at $f_{r}^{\lambda=-5}=300 \mathrm{~Hz}$ and $f_{r}^{\lambda=7}=-300 \mathrm{~Hz}$ in the rotor referential, which are actually overlapped on the FFT figure.

Naturally, the SDM with "resistance-limited" assumption $\left(N_{\lambda}=1\right)$ finds null rotor bar currents because the diffusion coefficient is the same for the fundamental of the stator mmf and for all the space harmonics, meaning $\overline{\alpha_{\lambda}}=\overline{\alpha_{1}}=0$ (as $s=$ 0 due to synchronism) for every $\lambda$.

The instantaneous torque is shown on Fig. 8. On the FFT, both SDM models in black and red curves seems to be overlapped but the time plot illustrates the harmonic discrepancies in terms of phase and magnitude. It is shown that the rotor mmf (only accounted for by the "skin-limited" approach) does not add new frequency content compared with the case without rotor $\mathrm{mmf}$ (for $N_{\lambda}=1$ ). Only phase and magnitude differ.

At no-load, the average torque - meaning the average value of the tangential force for $(f, r)=(0 H z, 0)-$ is null. Torque 


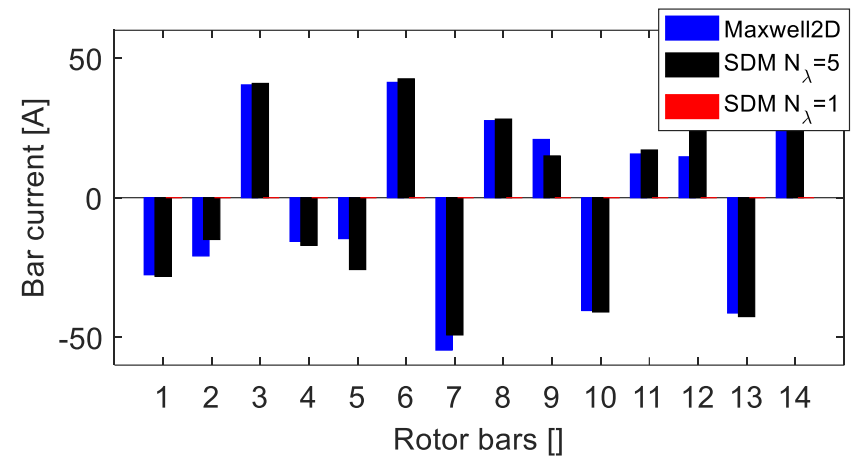

Fig. 6 Current distribution in rotor bars ( $\left(1^{\text {st }}\right.$ time step) at no-load
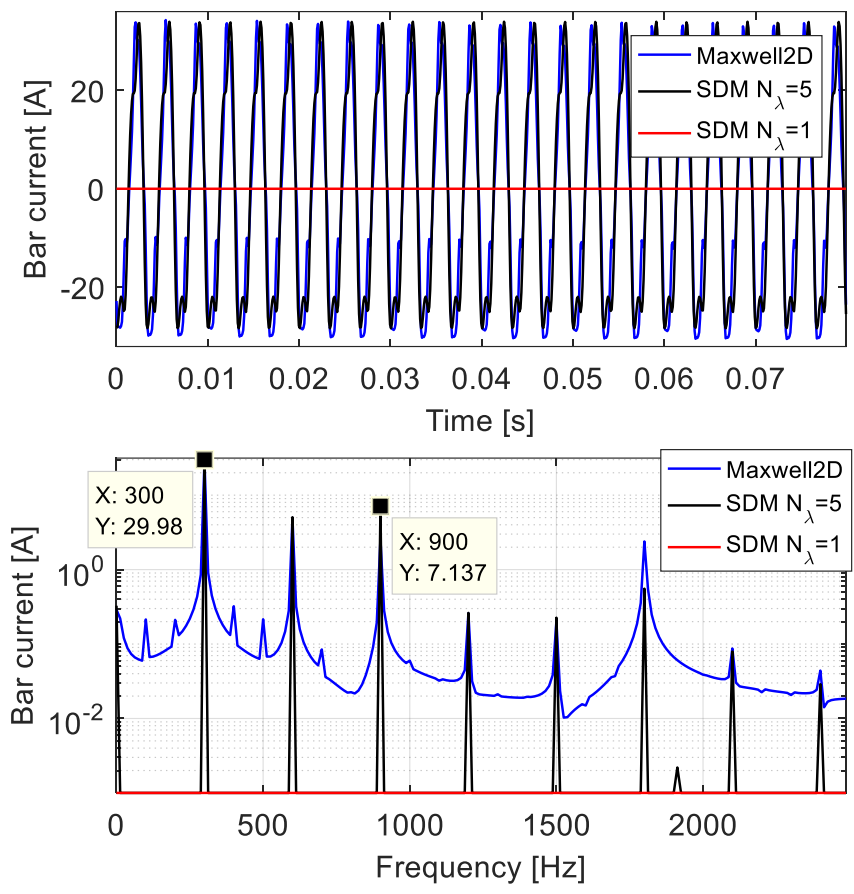

Fig. 7 Current flowing in the $1^{\text {st }}$ rotor bar over time at no-load and FFT.

ripple harmonics are in fact pulsating harmonics of tangential force whose orders are $(f>0, r=0)$. Using the convolution product theorem, each harmonic of force can be decomposed into a product of two flux density harmonics [17]. In this particular case, the first torque ripple harmonic $(600 \mathrm{~Hz}, 0)$ comes from the combination of two flux density harmonics $h_{1}$ and $h_{2}$ due to rotor slotting and $\mathrm{mmf}$ at:

$$
\begin{aligned}
& \left.\begin{array}{l}
h_{1}:\left(-f_{s},-Z_{r}+p\right) \\
h_{2}:\left(\left(\frac{Z_{r}}{p}(1-s)-1\right) f_{s}, Z_{r}-p\right)
\end{array}\right\} \stackrel{h_{1} * h_{2}}{=}\left(\left(\frac{Z_{r}}{p}(1-s)-2\right) f_{s}, 0\right) \\
& =(600 \mathrm{~Hz},
\end{aligned}
$$

The EMF is shown on Fig. 9. Time harmonics induced in the stator windings are due to the RSH given by equation (60). The EMF fundamental is well estimated by the three methods but the harmonic content is more accurate with the new $\operatorname{SDM}\left(N_{\lambda}=\right.$ 5 ) in terms of magnitude and phase.

\section{3) Conclusions for no-load condition $(s=0)$}

For this particular machine, the SDM with $N_{\lambda}=5$ is in very good agreement with the transient FEA. It verifies the assumption of constant skin effect for space harmonics higher
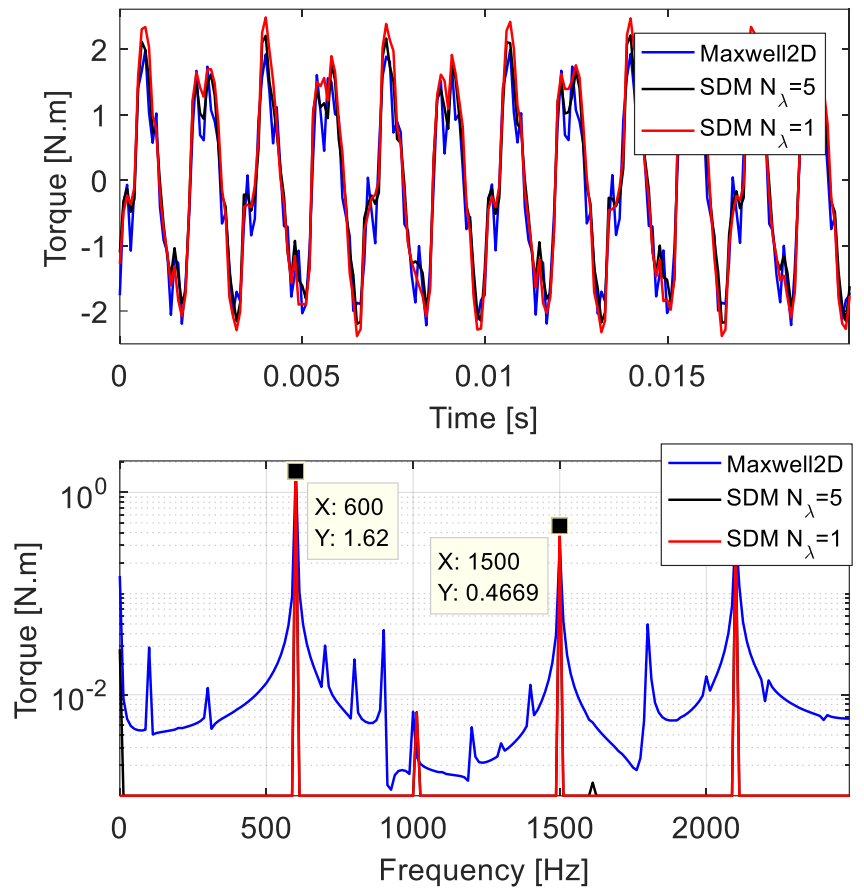

Fig. 8 Instantaneous torque over time at no-load and FFT.
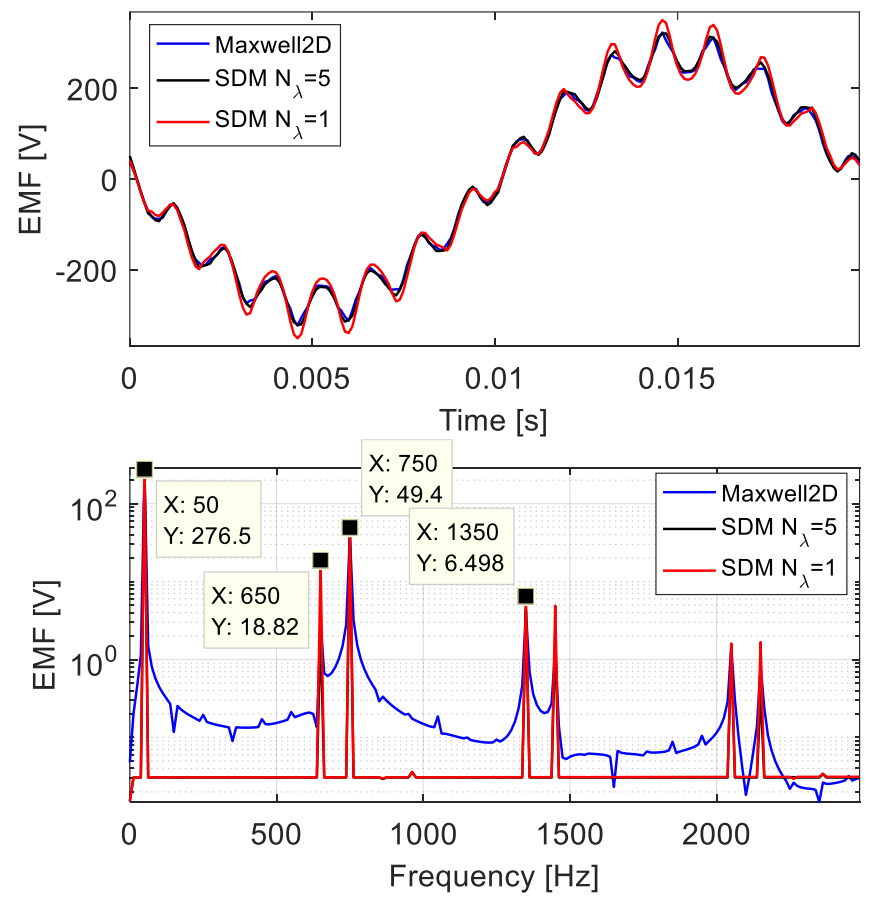

Fig. 9 EMF induced in the $1^{\text {st }}$ stator phase at no-load and FFT.

than the fifth one $(\lambda>13$, after equation (59)). For each electromagnetic quantity, both SDM with $N_{\lambda}=5$ ("skinlimited") and $N_{\lambda}=1$ ("resistance-limited") predict the same frequency content. In fact, the frequency values only depend on the rotor motion and the current sheet frequency, which are identical whether $N_{\lambda}=5$ or $N_{\lambda}=1$. However, SDM with $N_{\lambda}=$ 5 gives better results than the one with $N_{\lambda}=1$ in terms of magnitude and phase, because it enables to include the rotor $\mathrm{mmf}$ due to the space harmonics of the stator mmf. Besides, using the SDM with $N_{\lambda}=5$ increases the computation time by almost 5 as shown on TABLE II. 
On every time FFT, the FEA spectrum contains other small harmonics which are not predicted by the SDM and may not seem physical regarding the theoretical analyses found in the literature. These might be numerical artifacts introduced by mesh or during the estimation of the induced current. Besides, the peak harmonic are much sharper in the spectra given by SDM than the ones given by FEA. For the same simulation parameters, FEA introduces much more spectral leakage and numerical noise which may compromise the accuracy of the local magnetic forces computation.

\section{Results at Load Case ( $s=10 \%)$}

\section{1) Radial and tangential air gap flux densities}

The resulting radial and tangential flux density distributions over space and time at load are shown on Fig. 10 to Fig. 12. Only a fourth of the rotor electrical period is shown on the time plots. The air gap flux density has considerably decreased due to the induced rotor mmf which almost cancels the fundamental of the stator mmf at this high slip value.

The spatial FFT on Fig. 10 shows that the harmonic of order $r=Z_{s}-\left(Z_{r}-p\right)=10$ is wrongly estimated by the "resistance-limited" approach $\left(N_{\lambda}=1\right)$. In fact, this harmonic is due to the interaction of the harmonic of rotor mmf at $r=$ $Z_{r}-p$ with the first harmonic of stator slotting at $r=Z_{s}$. Because of the "resistance-limited" approach, an error is made on the rotor bar currents computation and consequently on the rotor mmf. This directly affects the $10^{\text {th }}$ spatial harmonic of the flux density and furthermore the harmonics of magnetic forces produced by this flux harmonic.

2) Bar currents, torque and Electromotive Force (EMF)

Rotor bar currents under load operation are shown on Fig. 13 and Fig. 14. The fundamental of the rotor currents is given by $f_{r}^{\lambda=1}=s f_{s}=5 \mathrm{~Hz}(\lambda=1$ is obtained for $\eta=0$ in equation (5)) and the first rotor current harmonics are at $f_{r}^{\lambda=-5}=275 \mathrm{~Hz}$ and $f_{r}^{\lambda=7}=-265 \mathrm{~Hz}$ in the rotor referential, from equation (8). In the stator referential, the frequency of these two rotor current harmonics are the same of the first PSH [18], at $f_{p s h}^{1}=$ $\{580 \mathrm{~Hz} ; 680 \mathrm{~Hz}\}$. In fact, rotor slotting and rotor mmf produce harmonics with the same time frequencies. From Fig. 14, it is shown that the fundamental of the rotor bar current is well estimated by the three methods but once again the harmonic content is more accurate with the new SDM for $N_{\lambda}=5$.

The same observations can be noted for both torque and EMF results at load state, respectively represented on Fig. 15 and Fig. 16. Furthermore, the obtained frequencies are matching with the theoretical equations.
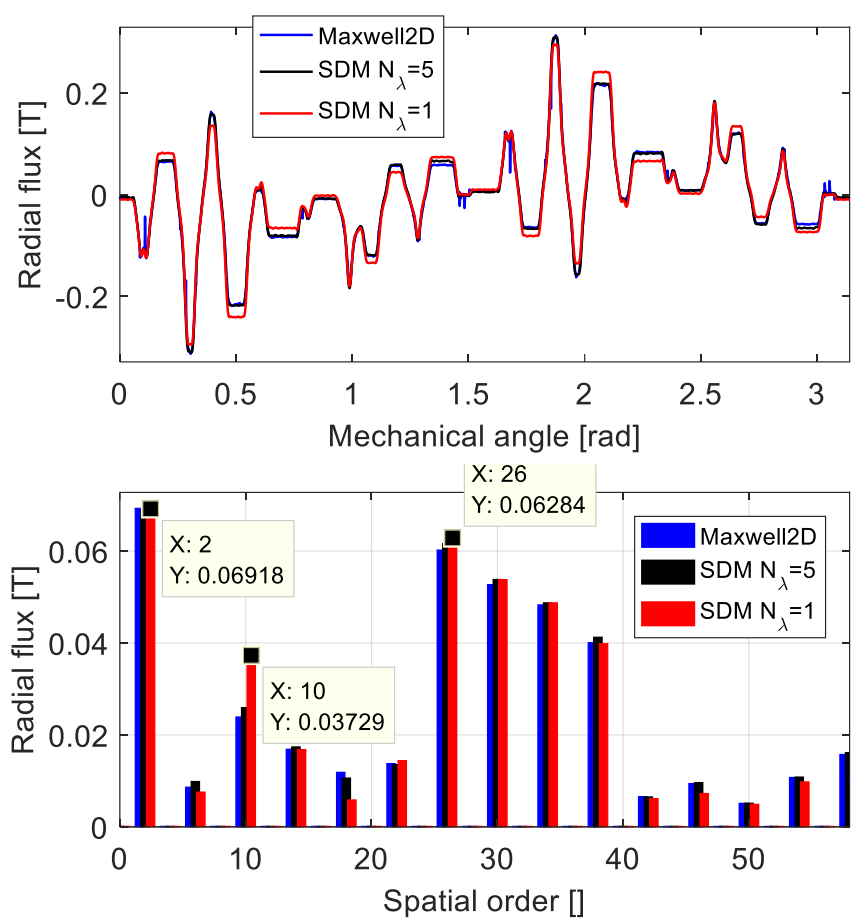

Fig. 10 Radial flux density over space ( $1^{\text {st }}$ time step) at load and FFT.
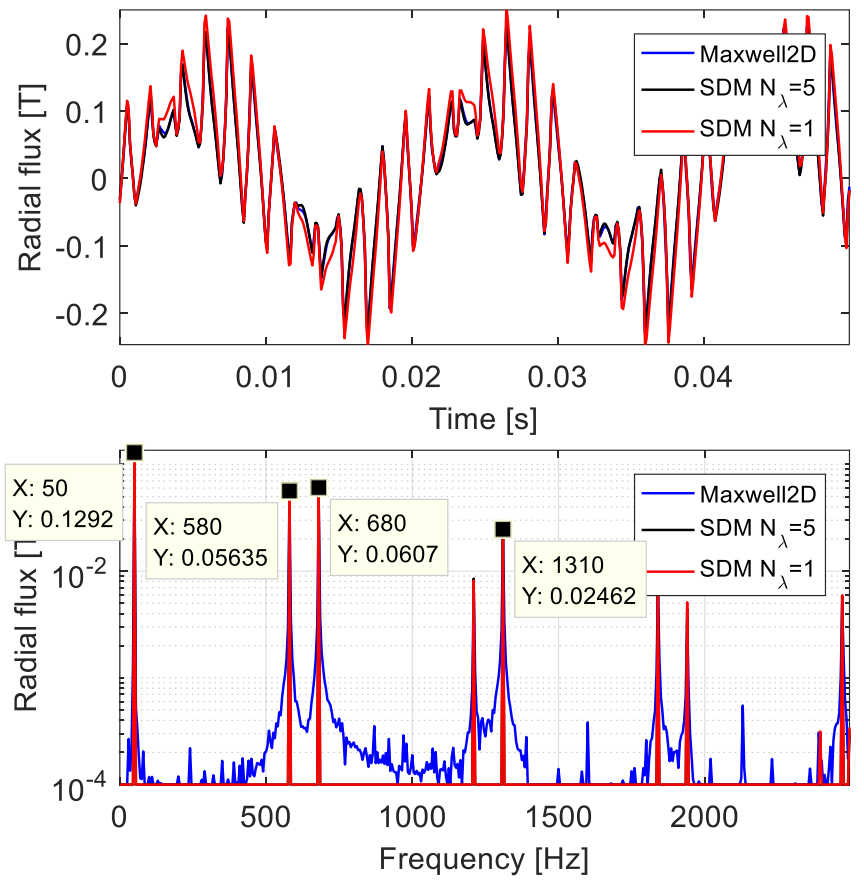

Fig. 11 Radial flux density over time at $\theta=0 \mathrm{rad}$ at load and FFT.

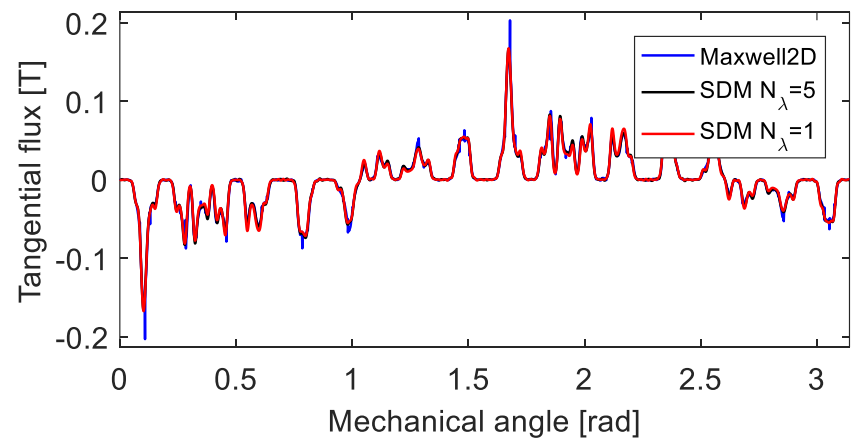

Fig. 12 Tangential flux density over space (first time step) at load. 


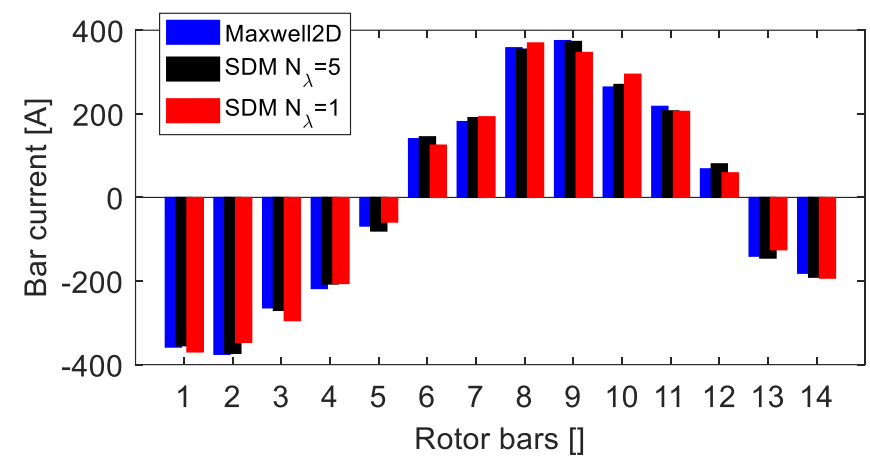

Fig. 13 Current distribution in rotor bars (first time step) at load
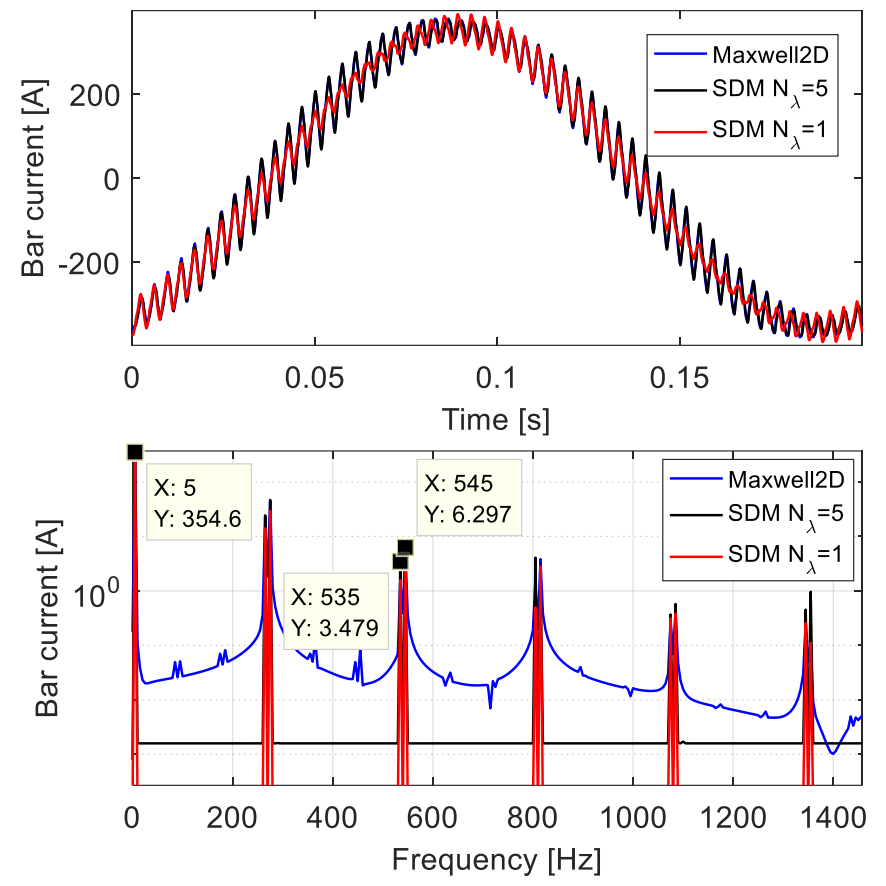

Fig. 14 Current flowing in the $1^{\text {st }}$ rotor bar over time at load

3) Conclusions for load case ( $s=10 \%$ )

As for the no-load case, the SDM with $N_{\lambda}=5$ is in very good agreement with the transient FEA under load operation. Despite a longer computation process, it is more accurate than the "resistance-limited" approach $\left(N_{\lambda}=1\right)$ to predict the harmonic content of every electromagnetic quantity in terms of phase and magnitude.

Besides, this simulation under load operation confirms the conclusions made previously on spectral leakage and numerical noise introduced by FEA.

\section{CONCLUSION}

In this paper, a new subdomain model is proposed for the simulation of squirrel cage induction machines at no-load and load state. This new model enables to accurately compute electromagnetic quantities such as air gap flux density, instantaneous torque and forces, electromotive force including slotting and mmf harmonic content. Compared with previous works, rotor slotting effects due to rotor motion is explicitly taken into account. Moreover, the skin effect in rotor bars is accurately modelled by considering each space harmonic of the stator magnetomotive force separately using current sheet
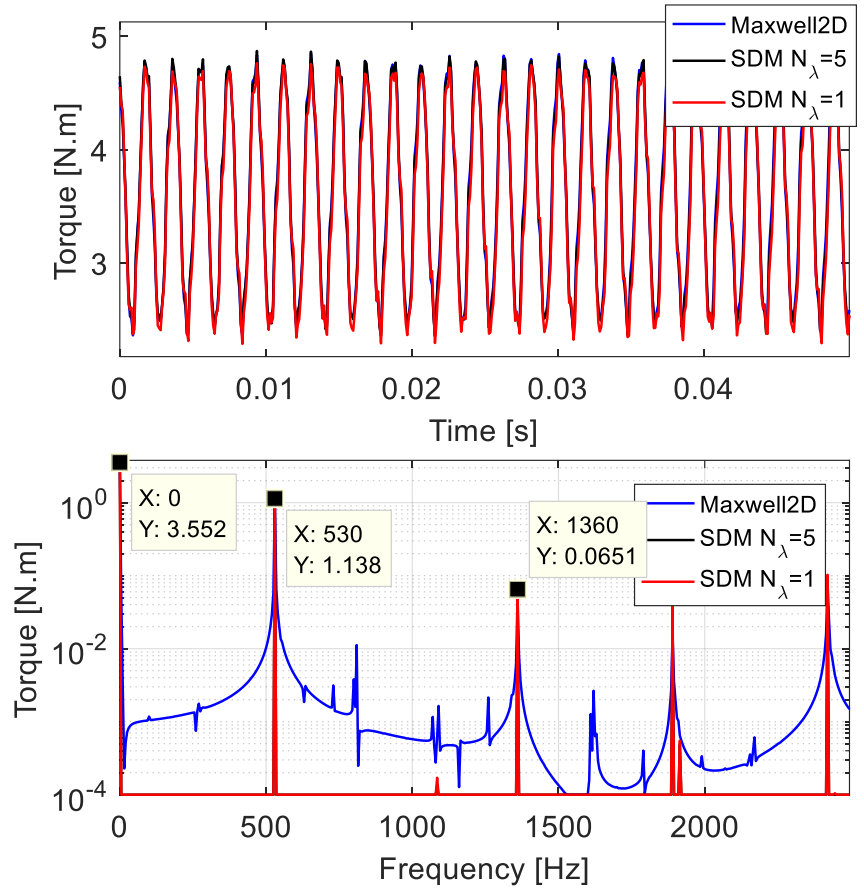

Fig. 15 Instantaneous torque (torque ripple) over time at load
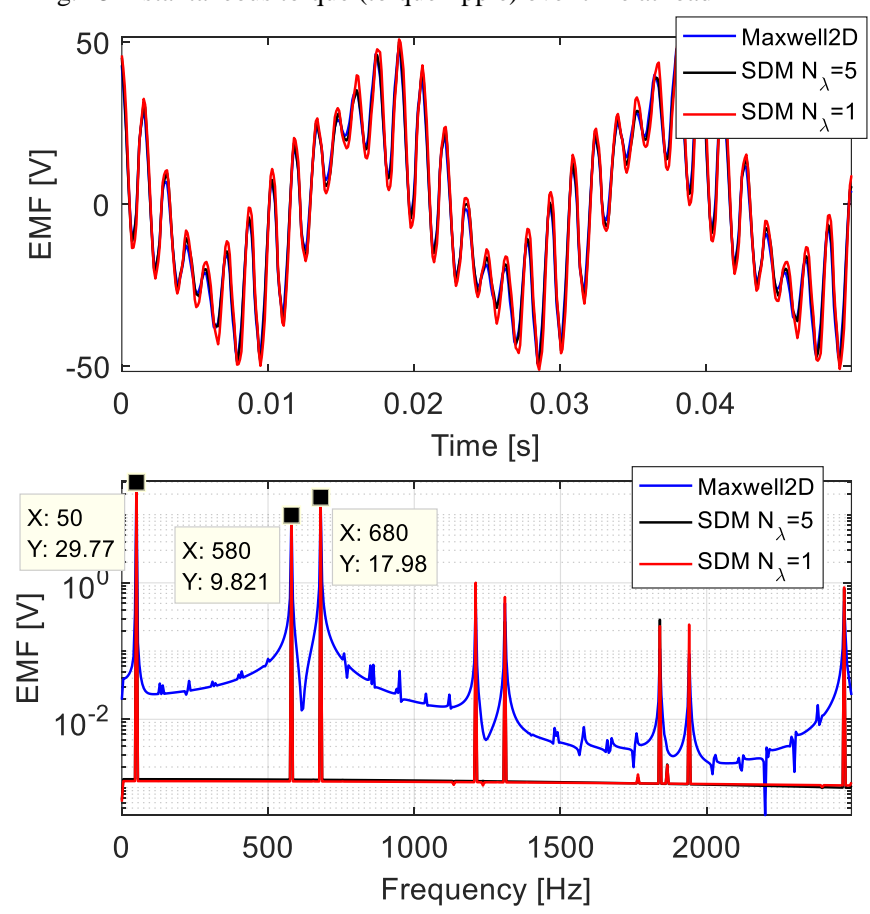

Fig. 16 EMF induced in the 1st stator phase over time at load

approach. Finally, the model directly gives the results at steadystate and so avoid numerical transient computation. The method have been validated with linear transient FEA and compared with the classical "resistance-limited" approach, showing better computational efficiency than FEA and better accuracy than the "resistance-limited" approach.

Therefore, the presented model is suited to magnetic force computation for vibroacoustic purposes. It has been integrated in MANATEE software [19] to study the effect of radial and tangential forces on a particular SCIM [4]. As a future work, it would be interesting to improve this SDM by adopting a 2D Fourier formulation in order to include the rotor slotting 
harmonics without using time-stepping technique. Another perspective could be using this model as an input to FEA to reduce the numerical transient computation time and further include magnetic saturation.

\section{REFERENCES}

$$
\text { Available: http://www.manatee-software.com. }
$$

Emile Devillers is currently doing an industrial $\mathrm{PhD}$ thesis at EOMYS ENGINEERING (Lille, France) and L2EP laboratory of the Ecole Centrale de Lille, North of France. His research interests are the modeling of noise and vibrations in electrical machines due to magnetic forces for reduction purposes. He obtained a M.Sc. specialized in Energy Systems in 2015 from the Grenoble Institute of Technology, France.

Jean Le Besnerais currently works in EOMYS ENGINEERING as an R\&D engineer on the analysis and reduction of acoustic noise and vibrations in electrical systems.

$\mathrm{He}$ made an industrial $\mathrm{PhD}$ thesis in Electrical Engineering at the L2EP laboratory of the Ecole Centrale de Lille, North of France, on the reduction of electromagnetic noise and vibrations in traction induction machines with ALSTOM Transport. In 2013, he founded EOMYS ENGINEERING, a company providing applied research and development services including modeling and simulation, scientific software development and experimental measurements.

Thierry Lubin received the M.Sc. degree from the University Pierre et Marie Curie, Paris 6, France in 1994 and the Ph.D. degree from the University Henri Poincaré, Nancy, France, in 2003. He is currently an associate professor of electrical engineering at the University of Lorraine, at the Groupe de Recherche en Electrotechnique et Electronique de Nancy (GREEN). His interests include analytical modeling of electrical devices, contactless torque transmission systems, modeling and control of synchronous reluctance motors, and applied superconductivity in electrical engineering.

Michel Hecquet received the Ph.D degree from the University of Lille, France, in 1995. His Ph.D dissertation presented a 3D permeance network of a claw-pole alternator, used for the simulation and the determination of the electromagnetic forces. Since 2008, he is full professor at Ecole Centrale de Lille in L2EP laboratory (Electrotechnic and Power Electronic Laboratory). His main interests are the development of multi-physics models of electrical machines (electromagnetic, mechanic and acoustic) and the optimal design of electrical machines.

Jean-Philippe Lecointe, DSc, received the MSc degree in Electrical Engineering from the Université des Sciences et Technologies de Lille, France, in 2000. He received the $\mathrm{PhD}$ degree from the Université d'Artois, France, in 2003. He is currently Full Professor at the Artois University and he is the head of the LSEE (Electrical Systems and Environment Research Laboratory), France. His research interests focus on efficiency, noise and vibrations of electrical machines. 